\title{
The effects of fast neutrons on inoperable carcinoma of the stomach
}

\author{
MARY CATTERALL, DEREK KINGSLEY, GILBERT LAWRENCE, \\ JOHN GRAINGER, AND JOHN SPENCER \\ From the Medical Research Council's Cyclotron Unit and the Departments of Radiodiagnosis, \\ Radiotherapy, Histopathology, and Surgery, the Royal Postgraduate Medical School and \\ Hammersmith Hospital, London
}

SUMMARY Thirty-nine unselected patients suffering from inoperable, recurrent, or residual adenocarcinoma of the stomach were referred for palliation with fast neutrons from the Medical Research Council's cyclotron at Hammersmith Hospital.

A full course of 1440 rads given in 12 treatments over 26 days was administered to the patients. Because of the relatively low energy $(7.5 \mathrm{MeV})$ of the beam from this particular machine, it was not possible to deliver the full dose uniformly throughout the tumour except in extremely thin patients.

Pain, dysphagia, vomiting, and bleeding were relieved in the majority of cases. The side effects were minimal and easily controlled. Palpable masses disappeared.

Five patients required surgery after neutron therapy. All the incisions were made through irradiated tissue and all except one healed normally. Tumour was present outside the treated area, but the absence of any palpable mass within the treated area was a consistent finding.

Radiologically, the stomachs remained abnormal and later changes included gross mucosal abnormality and shrinkage.

Fourteen patients came to necropsy and in 10 no tumour was present macroscopically. Tumour cells were seen in all except two cases but these were few, surrounded by dense fibrous tissue, and may not have been viable. The remaining stomach was abnormal with a thickened wall and destruction of mucosa. Three of the four cases in which macroscopic tumour was present received less than the standard dose because of the inadequate penetration of the beam.

Excellent regression of tumours was achieved by the neutrons, but the stomachs did not recover from this satisfactorily. Gastrectomy four to six months after treatment is therefore suggested. This operation and other surgical procedures in three other patients were successfully carried out.

There is a need for higher energy neutrons to improve treatment and extend it to patients of thick-set build.

Carcinoma of the stomach is one of the commonest malignant tumours in most countries of the world and in England and Wales the disease is the cause of 16000 deaths per annum. Burn (1971), in a prospective study of cases of gastric cancer referred to a group of European surgeons, found an overall oneyear survival rate of $24 \%$ and a five-year survival rate of only $8 \%$. Operative mortality was $18 \%$ after resection and $33 \%$ after total gastrectomy. Despite these poor results surgery still remains the main method of treating cancer of the stomach.

Received for publication 28 November 1974.
Radiotherapy has been used mainly as a palliative procedure (Guttmann, 1955). Nordman and Kauppinen (1972) and Wieland and Hymmen (1970) treated selected patients with doses of cobalt radiation of 3000 to 5000 rads and reported improved survival rates with relief of obstruction and haemorrhage. Several authors (Bowers and Brick, 1947; Brick, 1955; Moss and Brand, 1969) described the relative sensitivity of the gastric mucosa to $x$ and gamma irradiation, with perforation in about $11 \%$ of cases and ulcer formation and gastritis occurring at $\mathbf{4 5 0 0}$ to 5000 rads. Adenocarcinomas, in general, require higher doses than this for their sterilization (Wieland 
and Hymmen, 1970), and it is because of this poor tolerance by the tissues and the vomiting induced that radiotherapy alone can only be palliative. Chemotherapy used in combination with radiation gives slightly better results than when either is used alone (Nordman and Kauppinen, 1972).

Fast neutrons are uncharged particles which interact with tissues in an entirely different way from $x$ and gamma rays. They were first used in the treatment of carcinoma of the stomach in 1944 and four of five patients survived eight months (Stone, 1948). However, because of the technical difficulties and lack of understanding of the radiobiological effects of fast neutrons, this treatment was terminated in 1948. More recently Eichhorn, Lessel, and Matschke (1974) have reported their experience of combining neutrons with gamma radiation from Cobalt 60 . Using neutrons for $30 \%$ of the total dose, they found no microscopical evidence of tumour at necropsy in four out of nine patients, while with gamma rays alone all cases showed tumour at necropsy.

From 1969, treatment with fast neutrons has been given by the Medical Research Council's cyclotron at Hammersmith Hospital to 400 patients with a wide variety of inoperable and radioresistant tumours (Catterall, 1974). The results have been encouraging and this paper describes the clinical, radiological, and pathological effects of 39 of these patients, who suffered from advanced carcinoma of the stomach and were referred for palliation of their symptoms.

\section{The Patients}

The patients' ages ranged from 32 to 82 years. Thirty-one were male and eight were female. They were unselected except that all had inoperable, recurrent, or residual carcinomas. Table I gives details of the reasons for referral for neutron therapy.

Adenocarcinoma was verified histologically in each patient, usually at laparotomy, but in five who had no surgery, tissue was obtained at necropsy or at gastroscopy. Six tumours were well differentiated

\begin{tabular}{|c|c|c|c|c|}
\hline & & & & $\begin{array}{l}\text { No. of } \\
\text { Cases }\end{array}$ \\
\hline Inoperable at laparotomy $\quad \ldots$ & & .. & & 16 \\
\hline \multicolumn{5}{|c|}{ Inoperable at laparotomy and tumour bypassed with } \\
\hline $\begin{array}{lllll}\text { tube } & \cdots & \cdots & \cdots & \ldots\end{array}$ & . & & . & 4 \\
\hline Inoperable at laparotomy and ga & $0-i$ & al a & & \\
\hline mosis performed $\quad \ldots \quad \ldots$ & .. & .. & . & 5 \\
\hline Gastrectomy (residual tumour) & .. & . & .. & 3 \\
\hline Gastrectomy (recurrence) $\quad$. & . & . & . & 6 \\
\hline Gastrectomy and biopsy only .. & . & . & .. & 5 \\
\hline Total (all unselected cases) .. & . & . & . & 39 \\
\hline
\end{tabular}

Table I Reasons for referral to neutron clinic and six others were less well differentiated. The remainder were undifferentiated and four were described as linitis plastica.

\section{Methods}

The patients were treated on the cyclotron three times weekly for four weeks and a total dose of 1440 rads was delivered to the volume of the tumour and adjacent nodes. The neutrons from this particular machine are less penetrating than $x$ rays from megavoltage machines, and three, four, or six neutron beams had to be arranged to converge on the tumour. Even so, many patients did not receive the full dose uniformly throughout the tumour. Figure 1 shows a typical dose distribution. Each treatment lasted about four minutes. Nausea, if it occurred, was treated with pyridoxine or metoclopramide. Barium meal examinations were performed before and at intervals after treatment.

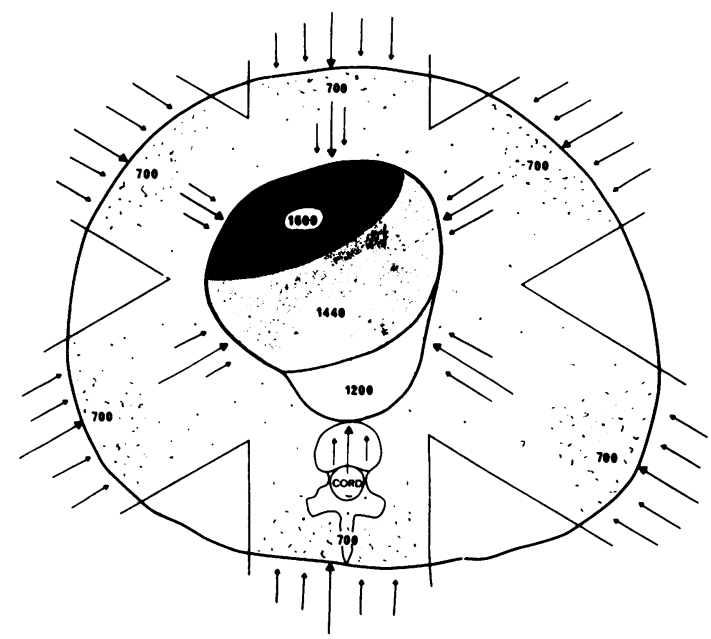

Fig 1 Transverse section of a patient with tumour involving the stomach and coeliac glands. Although six beams of radiation were used, they still resulted in an uneven dose distribution varying from 1600 to 1200 throughout the tumour volume.

\section{Results}

Of these 39 advanced cases, seven did not complete treatment and have been excluded. The results of treatment on the remaining 32 are given in table II.

In 18 of 20 patients, pain was relieved but recurred in two. The four patients with either persistent or recurrent pain had all had an oesophageal tube inserted. Dysphagia was completely relieved in 13 of 18 patients. Fourteen patients were vomiting before 


\begin{tabular}{llll}
\hline Symptom or Sign & Nos. Affected & Relief & Recurred \\
\hline Loss of appetite & 32 & 18 & 5 \\
Loss of weight & 28 & 9 & \\
Pain & 20 & 18 & 2 \\
Dysphagia & 18 & 13 & \\
Vomiting & 14 & 13 & \\
Palpable mass & 19 & 16 & \\
Severe bleeding & 6 & 6 & 1 \\
\hline
\end{tabular}

Table II Clinical effects of treatment ${ }^{1}$

Excluding seven patients who did not complete treatment.

treatment and this was stopped in all but one. Seven other patients vomited during treatment but not severely enough to interrupt the course.

Nineteen patients had a palpable epigastric mass which disappeared completely in 16 of them. Severe bleeding was stopped in all six patients, but it recurred four months after treatment in one case.

The mean survival time was $5 \cdot 1$ months, and all but two patients died of generalized metastases. One patient survived for two years, and three patients are alive five, six, and seven months after treatment.

SURGERY FOLLOWING NEUTRON THERAPY Five patients required surgery after radiotherapy: one for removal of a Celestin tube which was causing pain and hiccups, one for gastric haemorrhage, and three for intestinal obstruction. All incisions were made through irradiated tissues and all wounds healed normally except in one patient in whom a gastrostomy was undertaken one week after irradiation for the removal of a Celestin tube; a fistula resulted from which the patient died.

At operation the significant finding in all five cases was the absence of any palpable tumour mass within the irradiated area. Other viscera appeared normal with no evidence of telangiectases, perforations, or strictures. In three of these patients, tumour was found outside the treated area (fig 2), and was the cause of the intestinal obstruction for which surgery was undertaken.

In one patient (A.T.), in whom a total gastrectomy was undertaken six months after treatment because of persistent bleeding and cachexia, well defined tissue planes were found as the dissection proceeded. Palpation revealed a friable stomach wall which perforated on dissection, but no tumour was visible or palpable. The anastomosis remained intact even though the structures had been irradiated. The resected stomach (fig 3 ) showed an oedematous haemorrhagic mucosa with multiple ulcers but no sign of tumour. The stomach wall was thickened, oedematous, and fibrotic and showed radiation changes. No tumour was found microscopically.

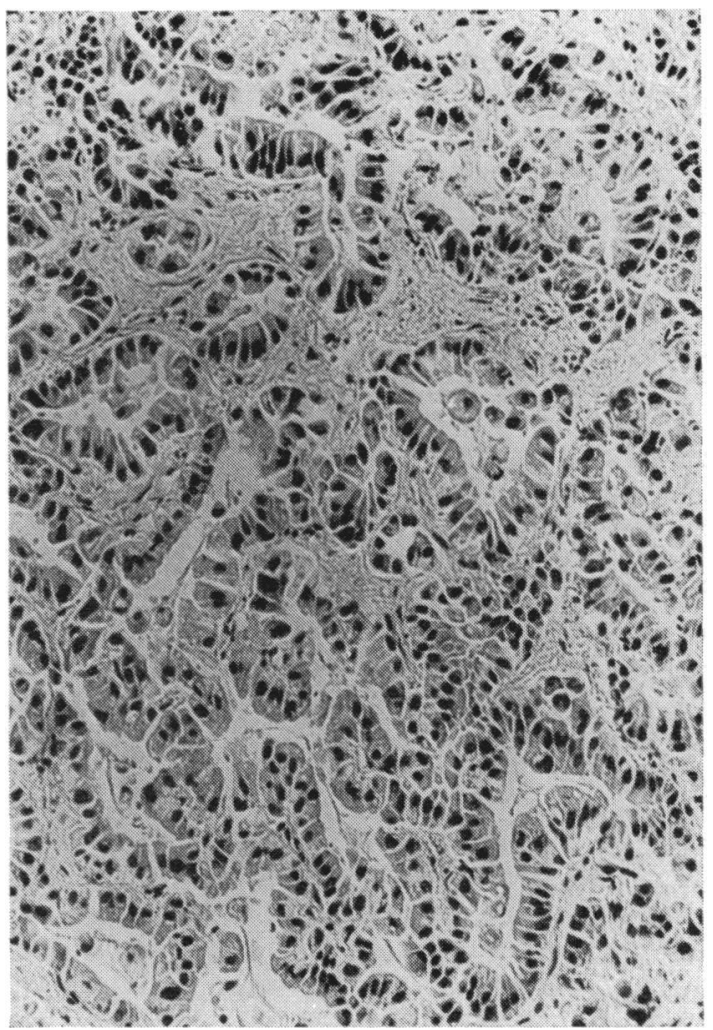

Fig 2 A moderately to well differentiated tumour which was outside the neutron-treated area. The part which was treated regressed completely.

\section{RADIOLOGICAL FINDINGS}

A barium meal examination was carried out on each patient before treatment. This was used in planning the size and position of the fields of treatment and was particularly valuable in cases where the mass of the tumour had been outlined with surgical clips. Following neutron therapy barium meals were undertaken at intervals of approximately two to three months. No barium meal showed reversion to a normal appearance. Interpretation of the radiographs was difficult and in most cases a number of follow-up examinations were required before any definite changes became established.

In the area of the tumour, changes in the mucosal pattern could not, by themselves, be attributed to the effects of neutrons since they could as easily be due to tumour infiltration. However, in those patients who had clips inserted round the tumour at laparotomy before treatment it was possible to assess regression of the tumour. By this method six out of seven patients showed a marked reduction in the size of the tumour after treatment.

In the region of normal stomach, marked changes 


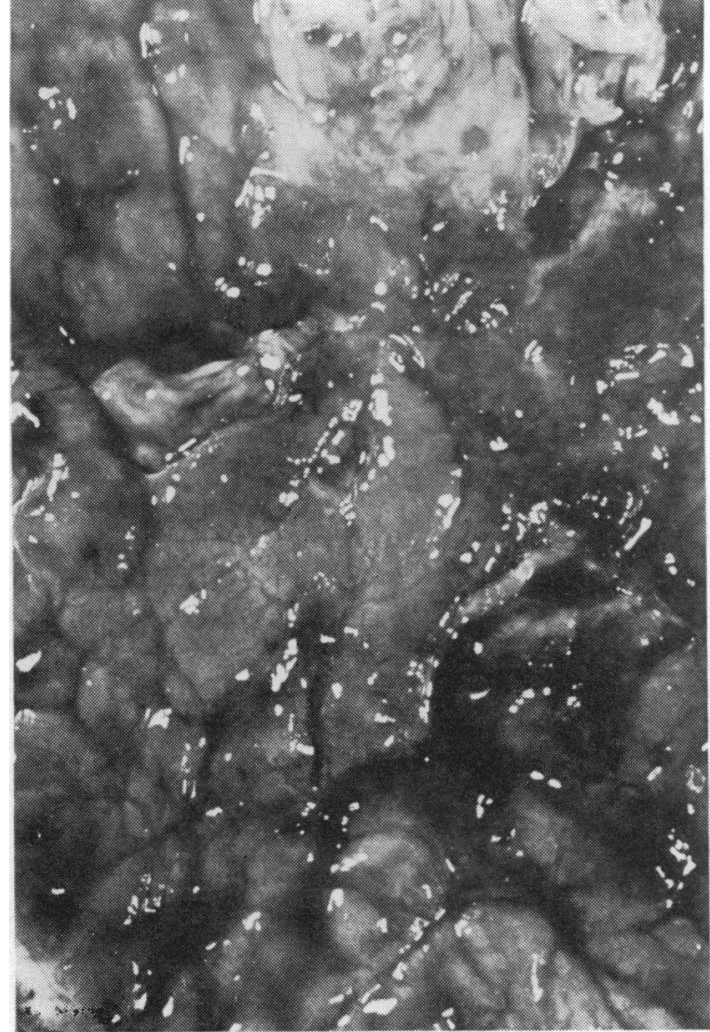

Fig 3 Close-up view of mucosa of gastrectomy specimen showing oedema, ulceration, and areas of haemorrhage but no tumour six months after neutron treatment. were seen. Gastric motility became reduced, and in patients who survived long enough there was no obvious peristaltic activity. In addition the size of the stomach showed a marked shrinkage (fig 4).

The mucosal appearance was the most difficult to assess. Sequential barium meals showed increasingly abnormal mucosal patterns. In some patients, particularly A.T. (fig 5), there was a very irregular polypoidal appearance which was thought at the time to be due to extension of the tumour. However, gastrectomy was performed six months after treatment and no macroscopic or microscopic tumour was found. Nine other patients who came to necropsy showed no sign of macroscopic tumour to support the changes seen radiologically.

A further assessment of these changes is, therefore, being made and will be the subject of a separate paper.

\section{NECROPSY FINDINGS}

In all, 14 patients came to necropsy (table III). Eleven of them had undergone laparotomy at which the tumour had been found to be so large, or was involving adjacent nodes and structures, that it was inoperable. In nine of these, however, only induration and no macroscopic tumour was present at necropsy, the causes of death being metastases in all but two patients. Two others, who still had a mass of tumour present, died only one and four weeks after the end of the treatment and one of them received less than the standard dose. Of the other three necropsies, two showed macroscopic tumour, but

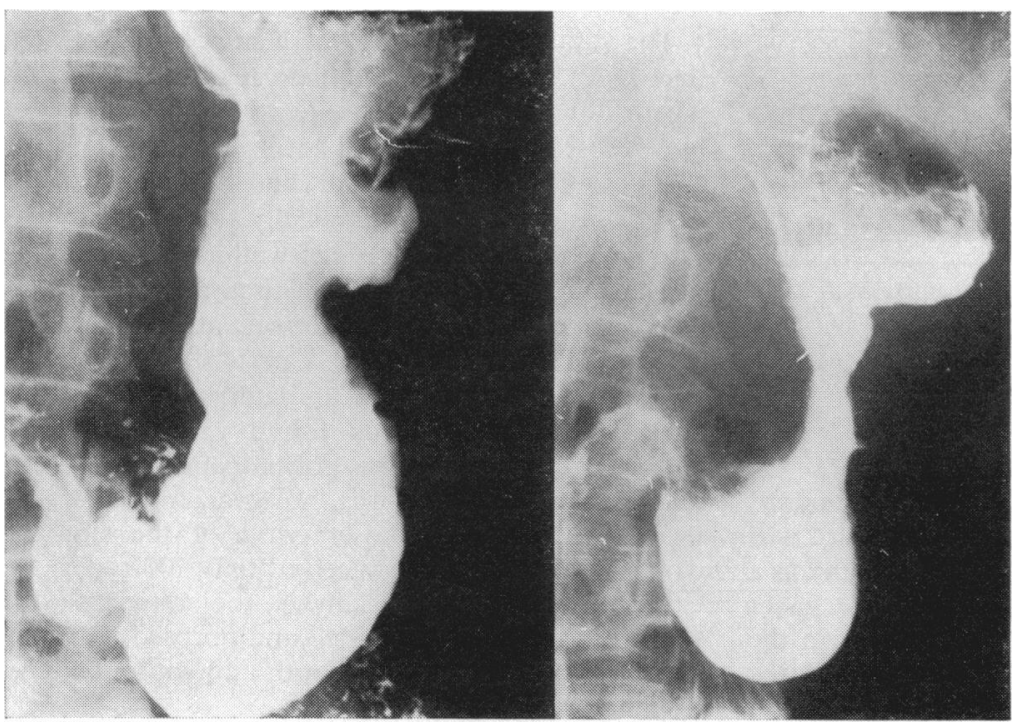

Fig 4 Barium meals before and 10 months after neutron therapy, showing marked shrinkage of the stomach. 


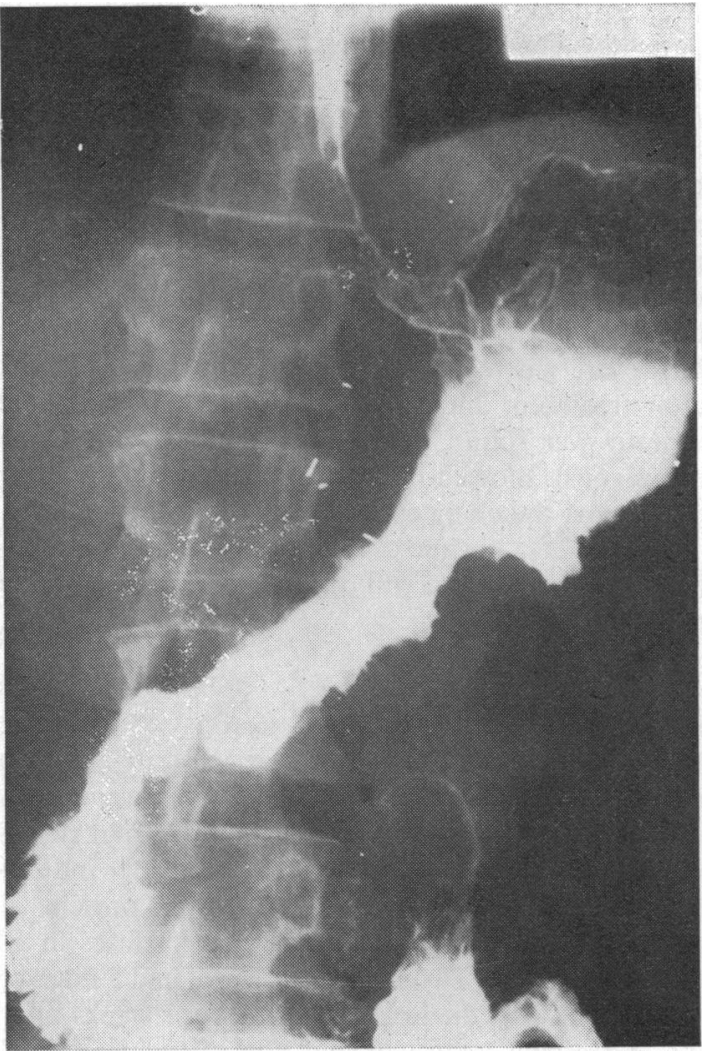

Fig 5

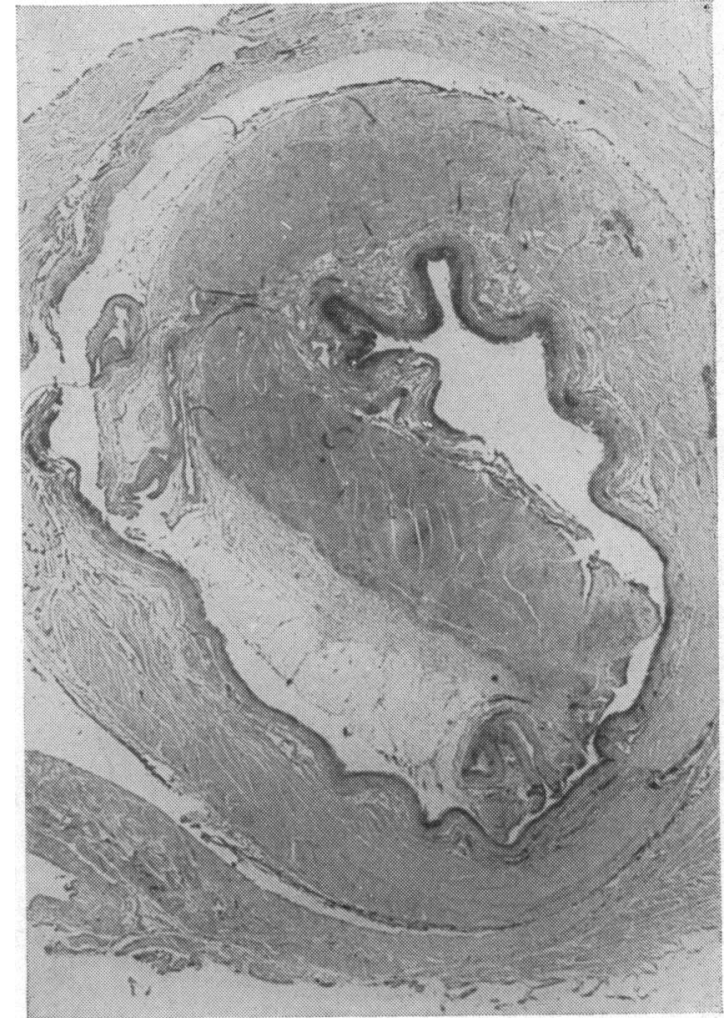

Fig 6

Fig 5 Appearance of barium meal six months after neutron therapy and one week before gastrectomy at which no tumour was found macroscopically or microscopically.

Fig 6 Longitudinal section through stomach showing no tumour where previously there was a bleeding cauliflower mass at gastroscopy.

\begin{tabular}{|c|c|c|c|c|c|}
\hline & & & & & $\begin{array}{l}\text { No. of } \\
\text { Cases }\end{array}$ \\
\hline Total $\quad . . \quad$. & .. & .. & .. & .. & 14 \\
\hline No gross tumour $\quad$.. & .. & .. & .. & .. & 10 \\
\hline Gross tumour present $\ldots$ & .. & .. & .. & .. & $4^{1}$ \\
\hline Contracted stomach $\quad \ldots$ & .. & .. & .. & .. & 11 \\
\hline Sealed gastric perforation & .. & .. & .. & .. & 2 \\
\hline
\end{tabular}

Table III Necropsy findings in the neutron-treated area

${ }^{1}$ Three of these received less than the standard dose and the other died four weeks after therapy (? inadequate time for tumour regression).

both of these patients received less than the standard dose -700 and 1200 rads respectively. The third was a patient whose tumour was seen at gastrectomy as a large cauliflower and bleeding mass on the lesser curvature. Although metastases were present in the liver, no tumour was found in the neutron-treated area (fig 6).
The response of one of these tumours illustrated that 1440 rads caused complete regression but that where 700 rads was received regression was followed by recurrence. This patient, following partial gastrectomy one year before, had recurrent tumour which was apparent radiologically at the pylorus and extended on to the abdominal wall as a bluish mass measuring $97 \times 65 \times 30 \mathrm{~mm}$. This part of the tumour received the full dose of neutrons and it regressed slowly but completely over a period of six months (fig 7). At necropsy two years later, no microscopic evidence of tumour was found on the anterior abdominal wall which was normal, apart from some telangiectasis. Tumour was, however, found around the coeliac axis and lesser curve of the stomach which, on analysis, had received only 700 rads.

In all except three specimens, the stomachs were small and the walls thickened and fibrosed. In most, the mucosal surface was smooth and without rugae. Two had sealed perforations. 


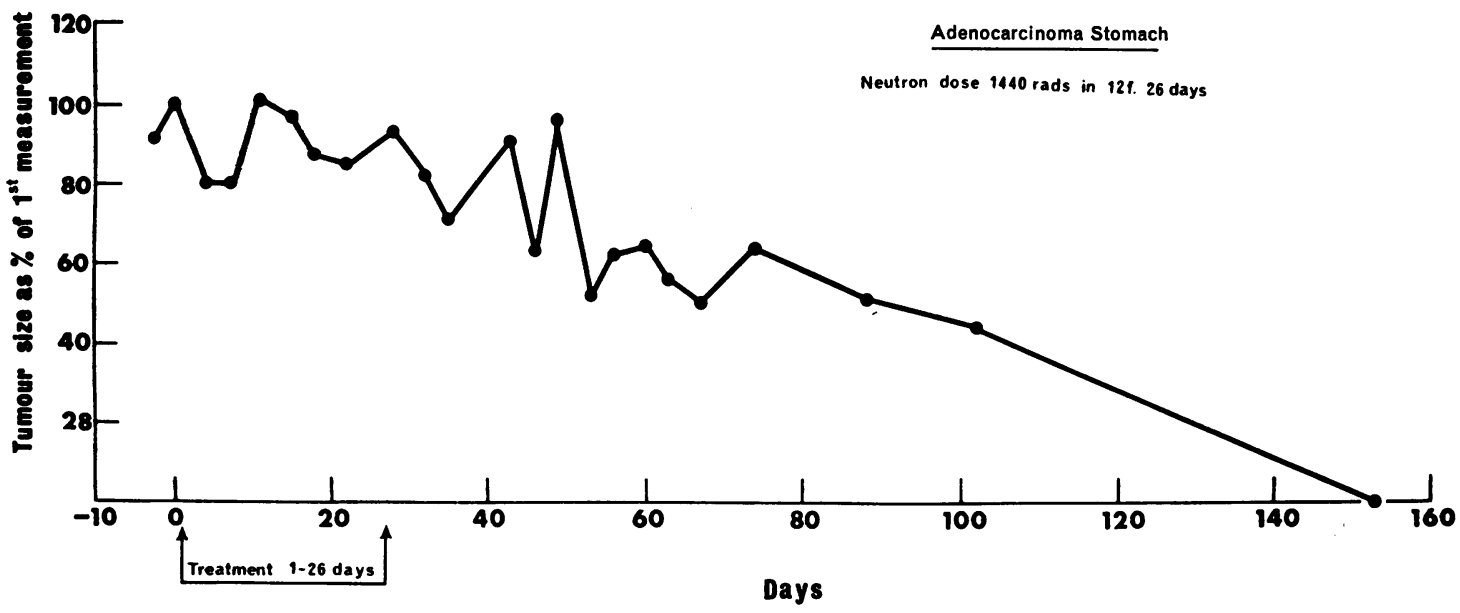

Fig 7 Serial measurements, expressed as percentages of the pretreatment tumour volume which was $97 \times 65 \times 30$ $\mathrm{mm}$, showing the slow but complete regression which took place.

Histologically, the thickening of the stomach walls was due to connective tissue reaction and fibrosis seen mainly in the submucosal and subserosal layers. Occasionally the muscle coat was partly or wholly replaced by fibrosis. Changes in the vessels were marked. There was eosinophilia, loss of endothelium, and occlusion of the lumen by thrombus or loose fibroblastic proliferation. Occasionally a vasculitis or apparent necrosis of the vessel wall was seen. In many sections, bacteria and fungi were visible in the mucosal and submucosal layers. Tumour cells were seen microscopically in all but two of the stomachs but were few in number in most cases and surrounded by dense fibrosis (fig 8).

\section{Discussion}

$X$ and gamma rays are seldom used in the treatment of gastric cancer because they have little effect on the tumour and they usually make the patient very sick. Fast neutrons caused the macroscopical disappearance of very large tumours at this site and did not cause serious sickness. The reasons for these findings are not yet understood. They are the more surprising in view of the relatively poor penetration of the beam which made the delivery of a uniform dose throughout the tumour volume impossible in most cases. At the same time, a greater amount of normal tissue was irradiated and this might have been expected to cause more, not less, vomiting.

Survival of the patient is usually the dominating factor in the assessment of any form of treatment. However, in a series of such advanced tumours as these, death frequently occurs from metastases even if the tumour has been controlled locally. This investigation was therefore undertaken to observe the systemic effects of neutron therapy on patients and assess its effects on the tumour and on adjacent

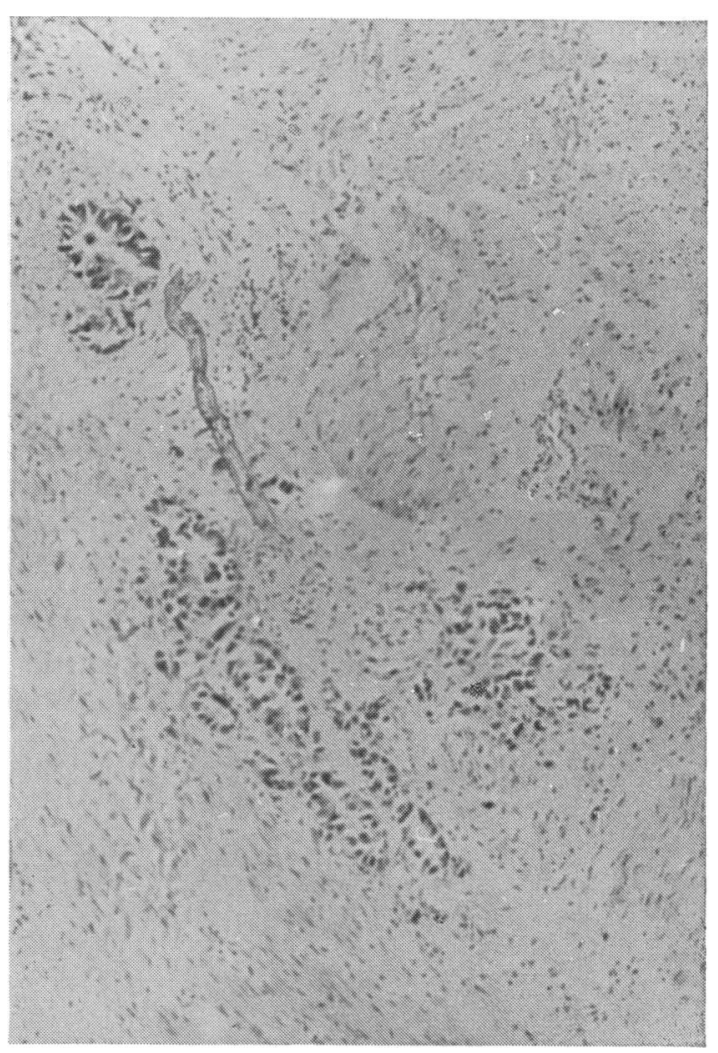

Fig 8 High-power view of stomach wall showing marked fibrosis surrounding adenocarcinoma cells. 
normal tissues. It was unreasonable to expect improved survival rates in these first unselected cases.

Symptomatic relief of dysphagia, vomiting, and pain was achieved in most patients and the insertion of an oesophageal tube before radiation is not recommended. This procedure caused pain in four of the five patients in whom it was performed. A nasogastric tube may be necessary to nourish the patient for the first week of treatment.

Objectively the effect of neutrons was satisfactory. Palpable masses regressed and macroscopical tumours were found at necropsy in only four cases, three of which had had an inadequate dose of neutrons and illustrated the importance of delivering the full 1440 rads to the whole tumour. Neutron beams of higher energy with greater penetration would deliver a uniform dose to the tumour, and, at the same time, the normal tissues which are unavoidably irradiated, would receive less radiation. Patients of thick-set build could also be treated, rather than only those who are thin.

The fact that complete macroscopic regression of large adenocarcinomas occurred seemed to us more significant than the persistence of a few residual tumour cells, although these were seen in all but two of the necropsies. Frequently the clumps of cells were small and in many cases were embedded in dense fibrous tissue within the wall of the stomach. These residual cells seemed to be very slow growing -almost inactive (Weinbren, personal communication). In only two cases were adjacent nodes found to contain tumour. This suggested a response in the node metastases similar to that of the primary tumour and agreed with the finding of Eichhorn et al (1974).

Following neutron therapy the stomach became a functionally useless cavity and a source of potential complications with sepsis and stasis contributing to the patient's general debility. However, the healing after surgery in all except one patient (whose operation was carried out only seven days after treatment) was encouraging and opens up possibilities of following a course of fast neutrons with surgical excision.

Gastrectomy following neutron therapy would have two main functions-the removal of any residual tumour cells and the elimination of a source of sepsis and a useless organ. An interval of four to six months between neutron therapy and gastrectomy is suggested for three reasons. It would give time for the tumour to regress, and the serial measurements shown in fig 7 illustrate how slow this can be. Secondly, it would allow the acute effects of radiation to subside. Thirdly, if occult metastases were present initially, they would become manifest and an inappropriate gastrectomy would be avoided.

This regime would be suitable for primary tumours of well or moderately well differentiated histological appearance, with direct extension to adjacent lymph nodes or to the liver, but not for discrete or separate metastases which could not be included in the treated volume.

In a disease which carries so lethal a prognosis there is evidence that the combined treatment of fast neutrons followed by total gastrectomy should be explored. Although it would be suitable only for selected cases, it could be a valuable contribution to the treatment of a commonly occurring tumour.

References

Bowers, R. F., and Brick, I. B. (1947). Surgery in radiation injury of the stomach. Surgery, 22, 20-40.

Brick, I. B. (1955). Effects of million volt irradiation on the gastrointestinal tract. Arch. intern. Med., 96, 26-31.

Burn, J. I. (1971). Cancer of stomach and oesophagus. Brit. J. Surg., $58,798-800$.

Catterall, M. (1974). The treatment of advanced cancer by fast neutrons from the Medical Research Council's Cyclotron at Hammersmith Hospital, London. Europ. J. Cancer, 10, 343-347.

Eichhorn, H. J., Lessel, A., and Matschke, S. (1974). Comparison between neutron therapy and ${ }^{\circ 0} \mathrm{Co}$ gamma ray therapy of bronchial, gastric and oesophageal carcinomata. Europ. J. Cancer, 10, 361-364.

Guttmann, R. J. (1955). Effect of 2 million volt Roentgen therapy on various malignant lesions of the upper abdomen. Amer. $J$. Roentgenol., 74, 204-212.

Moss, W. T., and Brand, W. N. (1969). Therapeutic Radiology, 3rd ed., pp. 276-294. Mosby, St. Louis.

Nordman, E., and Kauppinen, C. (1972). The value of megavolt therapy in carcinoma of the stomach. Strahlentherapic, 144, 635-640.

Stone, R. S. (1948). Neutron therapy and specific ionisation. Amer. J. Roentgenol., 59, 771-785.

Wieland, C., and Hymmen, U. (1970). Megavolttherapie maligner Neoplasien des Magens. Strahlentherapie, 140, 20-26.

Weinbren, K. (1974). Personal communication. 